\title{
ON THE MONOTONICITY OF $q$-SCHURER-STANCU TYPE POLYNOMIALS
}

\author{
ANA MARIA ACU, CARMEN VIOLETA MURARU, \\ AND VOICHIŢA ADRIANA RADU
}

Received 05 October, 2015

\begin{abstract}
Some properties of monotonicity and convexity of the q-Schurer -Stancu operators are considered. The paper contains also numerical examples based on Matlab algorithms, which verify these properties.
\end{abstract}

2010 Mathematics Subject Classification: 41A10; 41A36

Keywords: generalized Schurer-Stancu operators, q-integers, monotonicity, convexity

\section{Preliminaries}

In the last decades, the application of q-calculus represents one of the most interesting areas of research in approximation theory. Lupaş [12] introduced in 1987 a q-type of the Bernstein operators and in 1997 another generalization of these operators based on q-integers was introduced by Phillips [16]. Their approximation properties were studied by Videnskii [18], N. Mahmudov [13], T. Acar and A. Aral [1] and O. Dalmanoglu [9, 10]. In time, many authors have been studied new classes of q-generalized operators $([2-4,6,7,17])$.

Before proceeding further, we mention some basic definitions and notations from q-calculus. For any fixed real number $q>0$, the q-integer $[k]_{q}$, for $k \in \mathbb{N}$ is defined as

$$
[k]_{q}= \begin{cases}\frac{1-q^{k}}{1-q}, & q \neq 1, \\ k, & q=1 .\end{cases}
$$

The q-factorial integer and the q-binomial coefficients are :

$$
\begin{gathered}
{[k]_{q} != \begin{cases}{[k]_{q}[k-1]_{q} \ldots[1]_{q},} & k=1,2, \ldots \\
1, & k=0,\end{cases} } \\
{\left[\begin{array}{l}
n \\
k
\end{array}\right]_{q}=\frac{[n]_{q} !}{[k]_{q} ![n-k]_{q} !},(n \geq k \geq 0) .}
\end{gathered}
$$


The q-analoque of $(x-a)_{q}^{n}$ is the polynomial

$$
(x-a)_{q}^{n}= \begin{cases}1, & \text { if } n=0, \\ (x-a)(x-q a) \ldots\left(x-q^{n-1} a\right), & \text { if } n \geq 1 .\end{cases}
$$

Let $p$ be a non-negative integer and let $\alpha, \beta$ be some real parameters satisfying the conditions $0 \leq \alpha \leq \beta$. In 2003, D. Bărbosu [8] introduced for any $f \in C[0,1+p]$ and $x \in[0,1]$ the Schurer-Stancu operators as follows

$$
S_{m, p}^{(\alpha, \beta)}(f, q, x)=\sum_{k=0}^{m+p} p_{m, k}(x) f\left(\frac{k+\alpha}{m+\beta}\right),
$$

where $p_{m, k}(x)=\left(\begin{array}{c}m+p \\ k\end{array}\right) x^{k}(1-x)^{m+p-k}$.

Recently, P.N. Agrawal, V. Gupta and A.S. Kumar [5] introduced the class of qSchurer-Stancu operators. For any $m \in \mathbb{N}, p$ a fixed non negative integer number and $\alpha, \beta$ some real parameters satisfying the conditions $0 \leq \alpha \leq \beta$, they constructed the class of generalized q-Schurer-Stancu operators

$$
\tilde{S}_{m, p}^{(\alpha, \beta)}: C[0,1+p] \rightarrow C[0,1]
$$

as follows

$$
\tilde{S}_{m, p}^{(\alpha, \beta)}(f, q, x)=\sum_{k=0}^{m+p} \tilde{p}_{m, k}(x) f\left(\frac{[k]_{q}+\alpha}{[m]_{q}+\beta}\right), x \in[0,1],
$$

where $\tilde{p}_{m, k}(x)=\left[\begin{array}{c}m+p \\ k\end{array}\right]_{q} x^{k}(1-x)_{q}^{m+p-k}$.

If $\alpha=\beta=0$ the above operators reduce to the Bernstein-Schurer operators introduced by Muraru in [14].

Lemma 1 ([5]). For the operators defined in (1.1) the following properties hold 1. $\tilde{S}_{m, p}^{(\alpha, \beta)}\left(e_{0}, q, x\right)=1$,

2. $\tilde{S}_{m, p}^{(\alpha, \beta)}\left(e_{1}, q, x\right)=\frac{\alpha}{[m]_{q}+\beta}+\frac{[m+p]_{q}}{[m]_{q}+\beta} x$,

3.

$$
\begin{gathered}
\tilde{S}_{m, p}^{(\alpha, \beta)}\left(e_{2}, q, x\right) \\
=\frac{\alpha^{2}}{\left([m]_{q}+\beta\right)^{2}}+\frac{[m+p]_{q}^{2}}{\left([m]_{q}+\beta\right)^{2}} x^{2}+\frac{2 \alpha[m+p]_{q} x}{\left([m]_{q}+\beta\right)^{2}}+\frac{[m+p]_{q} x(1-x)}{\left([m]_{q}+\beta\right)^{2}} .
\end{gathered}
$$

The next result is based on Popoviciu's technique and it is expressed in terms of the first order modulus of continuity. 
Theorem 1 ([5]). If $f \in C[0,1+p]$ and $q \in(0,1)$ then

$$
\left\|\tilde{S}_{m, p}^{(\alpha, \beta)}(f, q, x)-f(x)\right\| \leq \frac{5}{4} \omega_{f}\left(\delta_{m}\right)
$$

holds, where

$$
\delta_{m}=\frac{1}{[m]_{q}+\beta} \sqrt{[m+p]_{q}+4\left(q^{m}[p]_{q}+\alpha-\beta\right)^{2}} .
$$

\section{MONOTONICITY OF THE $q$-SCHURER-STANCU OPERATORS}

Oruc and Philips [15] showed that for a convex function $f$ on [0,1], the q-Bernstein polynomials are monotonic decreasing. In this section we will prove a similar result for $q$-Schurer-Stancu operators.

Theorem 2. Let $f$ be a convex and increasing function on $[0, p+1]$. Then, for $0<q \leq 1$ and $\beta \leq \frac{[p]_{q}}{q^{p}}$,

$$
\tilde{S}_{m-1, p}^{(\alpha, \beta)}(f, q, x) \geq \tilde{S}_{m, p}^{(\alpha, \beta)}(f, q, x),
$$

for $0 \leq x \leq 1$ and $m \geq 2$.

Proof. For $0<q<1$ we have

$$
\begin{gathered}
\prod_{s=0}^{m+p-1}\left(1-q^{s} x\right)^{-1}\left[\tilde{S}_{m-1, p}^{(\alpha, \beta)}(f, q, x)-\tilde{S}_{m, p}^{(\alpha, \beta)}(f, q, x)\right] \\
=\sum_{k=0}^{m+p-1}\left[\begin{array}{c}
m+p-1 \\
k
\end{array}\right]_{q} x^{k} \prod_{s=m+p-k-1}^{m+p-1}\left(1-q^{s} x\right)^{-1} f\left(\frac{[k]_{q}+\alpha}{[m-1]_{q}+\beta}\right) \\
-\sum_{k=0}^{m+p}\left[\begin{array}{c}
m+p \\
k
\end{array}\right]_{q} x^{k} \prod_{s=m+p-k}^{m+p-1}\left(1-q^{s} x\right)^{-1} f\left(\frac{[k]_{q}+\alpha}{[m]_{q}+\beta}\right) .
\end{gathered}
$$

Denote

$$
\psi_{k}(x)=x^{k} \prod_{s=m+p-k}^{m+p-1}\left(1-q^{s} x\right)^{-1}
$$

and using the following relation

$$
x^{k} \prod_{s=m+p-k-1}^{m+p-1}\left(1-q^{s} x\right)^{-1}=\psi_{k}(x)+q^{m+p-k-1} \psi_{k+1}(x)
$$

we find

$$
\prod_{s=0}^{m+p-1}\left(1-q^{s} x\right)^{-1}\left[\tilde{S}_{m-1, p}^{(\alpha, \beta)}(f, q, x)-\tilde{S}_{m, p}^{(\alpha, \beta)}(f, q, x)\right]
$$




$$
\begin{gathered}
=\sum_{k=0}^{m+p-1} f\left(\frac{[k]_{q}+\alpha}{[m-1]_{q}+\beta}\right)\left[\begin{array}{c}
m+p-1 \\
k
\end{array}\right]_{q}\left\{\psi_{k}(x)+q^{m+p-k-1} \psi_{k+1}(x)\right\} \\
-\sum_{k=0}^{m+p} f\left(\frac{[k]_{q}+\alpha}{[m]_{q}+\beta}\right)\left[\begin{array}{c}
m+p \\
k
\end{array}\right]_{q} \psi_{k}(x)=\sum_{k=0}^{m+p-1} f\left(\frac{[k]_{q}+\alpha}{[m-1]_{q}+\beta}\right)\left[\begin{array}{c}
m+p-1 \\
k
\end{array}\right]_{q} \psi_{k}(x) \\
+\sum_{k=1}^{m+p} q^{m+p-k} f\left(\frac{[k-1]_{q}+\alpha}{[m-1]_{q}+\beta}\right)\left[\begin{array}{c}
m+p-1 \\
k-1
\end{array}\right]_{q} \psi_{k}(x) \\
-\sum_{k=0}^{m+p} f\left(\frac{[k]_{q}+\alpha}{[m]_{q}+\beta}\right)\left[\begin{array}{c}
m+p \\
k
\end{array}\right]_{q} \psi_{k}(x)=\sum_{k=1}^{m+p-1}\left\{f\left(\frac{[k]_{q}+\alpha}{[m-1]_{q}+\beta}\right)\left[\begin{array}{c}
m+p-1 \\
k
\end{array}\right]_{q}\right. \\
\left.+q^{m+p-k} f\left(\frac{[k-1]_{q}+\alpha}{[m-1]_{q}+\beta}\right)\left[\begin{array}{c}
m+p-1 \\
k-1
\end{array}\right]_{q}-f\left(\frac{[k]_{q}+\alpha}{[m]_{q}+\beta}\right)\left[\begin{array}{c}
m+p \\
k
\end{array}\right]_{q}\right\} \psi_{k}(x) \\
+\left\{f\left(\frac{[m+p-1]_{q}+\alpha}{[m-1]_{q}+\beta}\right)-f\left(\frac{[m+p]_{q}+\alpha}{[m]_{q}+\beta}\right)\right\} \psi_{m+p}(x) \\
+\left\{f\left(\frac{\alpha}{[m-1]_{q}+\beta}\right)-f\left(\frac{\alpha}{[m]_{q}+\beta}\right)\right\} \psi_{0}(x) \\
=\sum_{k=1}^{m+p-1}\left[\begin{array}{c}
m+p \\
k
\end{array}\right]_{q}^{a_{k} \psi_{k}(x)+\left\{f\left(\frac{[m+p-1]_{q}+\alpha}{[m-1]_{q}+\beta}\right)-f\left(\frac{[m+p]_{q}+\alpha}{[m]_{q}+\beta}\right) \psi_{m+p}(x)\right.} \\
+\left\{f\left(\frac{\alpha}{[m-1]_{q}+\beta}\right)-f\left(\frac{\alpha}{[m]_{q}+\beta}\right)\right\} \psi_{0}(x),
\end{gathered}
$$

where

$$
\begin{aligned}
a_{k}= & f\left(\frac{[k]_{q}+\alpha}{[m-1]_{q}+\beta}\right) \frac{[m+p-k]_{q}}{[m+p]_{q}}+q^{m+p-k} f\left(\frac{[k-1]_{q}+\alpha}{[m-1]_{q}+\beta}\right) \frac{[k]_{q}}{[m+p]_{q}} \\
& -f\left(\frac{[k]_{q}+\alpha}{[m]_{q}+\beta}\right) .
\end{aligned}
$$

From (2.2) it is clear that each $\psi_{k}(x)$ is non-negative on $[0,1]$ for $0 \leq q \leq 1$ and thus, it suffices to show that each $a_{k}$ is non-negative.

Since $f$ is convex on $[0, p+1]$, for any $t_{0}, t_{1}$ such that $0 \leq t_{0}<t_{1} \leq p+1$ and any $\lambda, 0<\lambda<1$, we have

$$
f\left(\lambda t_{0}+(1-\lambda) t_{1}\right) \leq \lambda f\left(t_{0}\right)+(1-\lambda) f\left(t_{1}\right) .
$$

Let $t_{0}=\frac{[k-1]_{q}+\alpha}{[m-1]_{q}+\beta}, t_{1}=\frac{[k]_{q}+\alpha}{[m-1]_{q}+\beta}$ and $\lambda=q^{m+p-k} \frac{[k]_{q}}{[m+p]_{q}}$. Then $0 \leq t_{0}<t_{1} \leq p+1$ and $0<\lambda<1$ for $1 \leq k \leq m+p-1$. If we replace them in the relation (2.3), it follows

$$
q^{m+p-k} \frac{[k]_{q}}{[m+p]_{q}} f\left(\frac{[k-1]_{q}+\alpha}{[m-1]_{q}+\beta}\right)+\frac{[m+p-k]_{q}}{[m+p]_{q}} f\left(\frac{[k]_{q}+\alpha}{[m-1]_{q}+\beta}\right)
$$




$$
\geq f\left(q^{m+p-k} \frac{[k]_{q}}{[m+p]_{q}} \cdot \frac{[k-1]_{q}+\alpha}{[m-1]_{q}+\beta}+\frac{[m+p-k]_{q}}{[m+p]_{q}} \cdot \frac{[k]_{q}+\alpha}{[m-1]_{q}+\beta}\right) .
$$

Using the inequality $[k]_{q}\left([k-1]_{q}+\alpha\right) \geq\left([k]_{q}+\alpha\right)[k-1]_{q}$ and $f$ increasing function, it follows

$$
\begin{aligned}
& q^{m+p-k} \frac{[k]_{q}}{[m+p]_{q}} f\left(\frac{[k-1]_{q}+\alpha}{[m-1]_{q}+\beta}\right)+\frac{[m+p-k]_{q}}{[m+p]_{q}} f\left(\frac{[k]_{q}+\alpha}{[m-1]_{q}+\beta}\right) \\
\geq & f\left(\frac{[k]_{q}+\alpha}{[m+p]_{q}} \cdot \frac{q^{m+p-k}[k-1]_{q}+[m+p-k]_{q}}{[m-1]_{q}+\beta}\right)=f\left(\frac{[k]_{q}+\alpha}{[m+p]_{q}} \cdot \frac{[m+p-1]_{q}}{[m-1]_{q}+\beta}\right) .
\end{aligned}
$$

Since $f$ is increasing on $[0, p+1]$ and

$$
\begin{gathered}
a_{k}=f\left(\frac{[k]_{q}+\alpha}{[m-1]_{q}+\beta}\right) \frac{[m+p-k]_{q}}{[m+p]_{q}}+q^{m+p-k} f\left(\frac{[k-1]_{q}+\alpha}{[m-1]_{q}+\beta}\right) \frac{[k]_{q}}{[m+p]_{q}} \\
-f\left(\frac{[k]_{q}+\alpha}{[m+p]_{q}} \cdot \frac{[m+p-1]_{q}}{[m-1]_{q}+\beta}\right)+\left\{f\left(\frac{[k]_{q}+\alpha}{[m+p]_{q}} \cdot \frac{[m+p-1]_{q}}{[m-1]_{q}+\beta}\right)-f\left(\frac{[k]_{q}+\alpha}{[m]_{q}+\beta}\right)\right\},
\end{gathered}
$$

from the inequality (2.4) we obtain $a_{k} \geq 0, k=\overline{1, m+p-1}$.

Therefore $\tilde{S}_{m-1, p}^{(\alpha, \beta)}(f, q, x) \geq \tilde{S}_{m, p}^{(\alpha, \beta)}(f, q, x)$.

For $q=1$ and $0 \leq x<1$ in a similar way the property (2.1) is verified.

For $q=1$ and $x=1$ we have

$$
\tilde{S}_{m-1, p}^{(\alpha, \beta)}(f, 1,1)-\tilde{S}_{m, p}^{(\alpha, \beta)}(f, 1,1)=f\left(\frac{m+p-1+\alpha}{m-1+\beta}\right)-f\left(\frac{m+p+\alpha}{m+\beta}\right) \geq 0 .
$$

Theorem 3. If $f$ is convex, then for all $m \geq 1$ and $0<q \leq 1$ it follows

i) $\tilde{S}_{m, p}^{(\alpha, \beta)}(f, q, x) \geq f(x)$, for $x \in[0,1], \quad f$ increasing on $[0,1]$ and $\beta=\alpha+\varepsilon, \varepsilon \in\left[0, q^{m}[p]_{q}\right]$

ii) $\tilde{S}_{m, p}^{(\alpha, \beta)}(f, q, x) \geq f(x)$, for $x \in\left[0, \frac{\alpha}{\beta-q^{m}[p]_{q}}\right]$, $f$ increasing on $[0,1]$ and $\beta>\alpha+q^{m}[p]_{q} ;$

iii) $\tilde{S}_{m, p}^{(\alpha, \beta)}(f, q, x) \geq f(x)$, for $x \in\left(\frac{\alpha}{\beta-q^{m}[p]_{q}}, 1\right]$, $f$ decreasing on $[0,1]$ and $\beta>\alpha+q^{m}[p]_{q}$. follows

Proof. We consider the knotes $x_{k}=\frac{[k]_{q}+\alpha}{[m]_{q}+\beta}, 0 \leq k \leq m+p$. From Lemma 1 it

$$
\sum_{k=0}^{m+p} \tilde{p}_{m, k}(x)=1, \sum_{k=0}^{m+p} \tilde{p}_{m, k}(x) x_{k}=\frac{\alpha}{[m]_{q}+\beta}+\frac{[m+p]_{q}}{[m]_{q}+\beta} x .
$$


Using the convexity of function $\mathrm{f}$ we have

$$
\begin{aligned}
\tilde{S}_{m, p}^{(\alpha, \beta)}(f, q, x) & =\sum_{k=0}^{m+p} \tilde{p}_{m, k}(x) f\left(x_{k}\right) \geq f\left(\sum_{k=0}^{m+p} \tilde{p}_{m, k}(x) x_{k}\right) \\
& =f\left(\frac{\alpha}{[m]_{q}+\beta}+\frac{[m+p]_{q}}{[m]_{q}+\beta} x\right) .
\end{aligned}
$$

The following inequalities hold
a) $\frac{\alpha}{[m]_{q}+\beta}+\frac{[m+p]_{q}}{[m]_{q}+\beta} x \geq x$ for $\beta=\alpha+\epsilon, \epsilon \in\left[0, q^{m}[p]\right], x \in[0,1]$;
b) $\frac{\alpha}{[m]_{q}+\beta}+\frac{[m+p]_{q}}{[m]_{q}+\beta} x \geq x$ for $\beta>\alpha+q^{m}[p]_{q}, x \in\left[0, \frac{\alpha}{\beta-q^{m}[p]_{q}}\right]$;
c) $\frac{\alpha}{[m]_{q}+\beta}+\frac{[m+p]_{q}}{[m]_{q}+\beta} x \leq x$ for $\beta>\alpha+q^{m}[p]_{q}, x \in\left[\frac{\alpha}{\beta-q^{m}[p]_{q}}, 1\right]$.

The theorem is proved using the monotony of function $f$ and the inequalities a)c).

\section{NUMERICAL EXAMPLE}

Davis [11] proved that for any convex function $f$, the classical Bernstein polynomial is convex and the sequence of Bernstein polynomials is monotonic decreasing. Oruc and Philips [15] extend these results for the Bernstein operators in $q$-calculus for $0<q \leq 1$. In this section we will verify numerically these properties for the q-Schurer-Stancu operators.

TABLE 1. The q-Schurer-Stancu operators

\begin{tabular}{|l|l|l|}
\hline$x$ & $\tilde{S}_{30,6}^{(3,5)}(f, q, x)$ & $\tilde{S}_{90,6}^{(3,5)}(f, q, x)$ \\
\hline 0 & 0.029115231597413 & 0.026565252311249 \\
\hline 0.1 & 0.089811163243826 & 0.083383934253406 \\
\hline 0.2 & 0.191687642176340 & 0.179475155093384 \\
\hline 0.3 & 0.347058215579779 & 0.326749960898829 \\
\hline 0.4 & 0.570050753689721 & 0.538887425606676 \\
\hline 0.5 & 0.876786656660820 & 0.831510780091276 \\
\hline 0.6 & 1.285573442130916 & 1.222376845885256 \\
\hline 0.7 & 1.817111513724159 & 1.731579581814080 \\
\hline 0.8 & 2.494715950321931 & 2.381768592612226 \\
\hline 0.9 & 3.344554197167635 & 3.198383491142964 \\
\hline 1 & 4.395900582819147 & 4.209905050209471 \\
\hline
\end{tabular}


In Table 1 are calculated the values of the q-Schurer-Stancu operators $\tilde{S}_{30,6}^{(3,5)}(f, q, x)$ and $\tilde{S}_{90,6}^{(3,5)}(f, q, x)$ for $f(x)=x^{3} e^{x+1}$ and $q=0.9$. Also, in the Figure 1 are given the graphics of these operators.

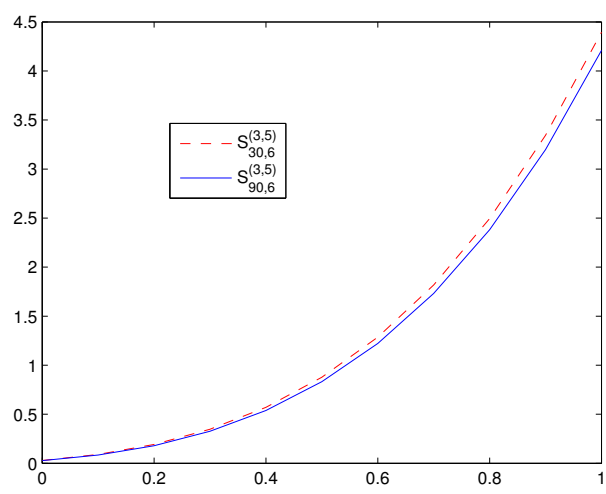

FIGURE 1 . The monotonicity of the $q$-Schurer-Stancu operators

In the next part of this section we will give some numerical examples which verify the inequalities proved in Theorem 3.

Example 1. If $n=50, p=5, \alpha=3, \beta=3.0211, q=0.9, f(x)=x^{3} e^{x+1}$, it follows $\tilde{S}_{m, p}^{(\alpha, \beta)}(f, q, x) \geq f(x)$ for all $x \in[0,1]$.

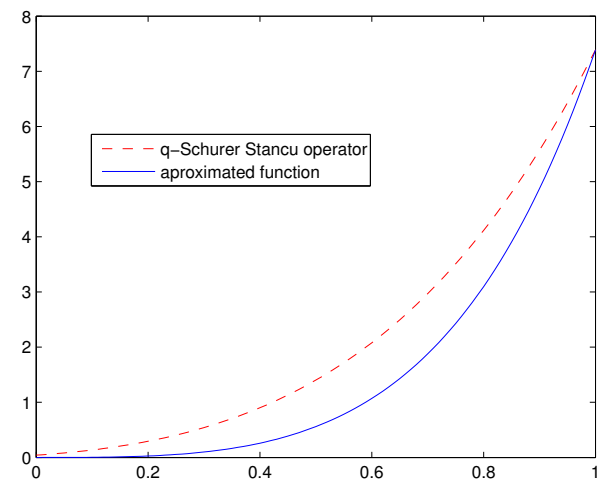

FIGURE 2. The $q$-Schurer-Stancu operators for increasing function and $\beta=\alpha+\epsilon$ 
Example 2. If $n=50, p=5, \alpha=3, q=0.9, \beta=8.0211, f(x)=x^{3} e^{x+1}$, it follows $\tilde{S}_{m, p}^{(\alpha, \beta)}(f, q, x) \geq f(x)$ for all $x \in[0,0.375]$.

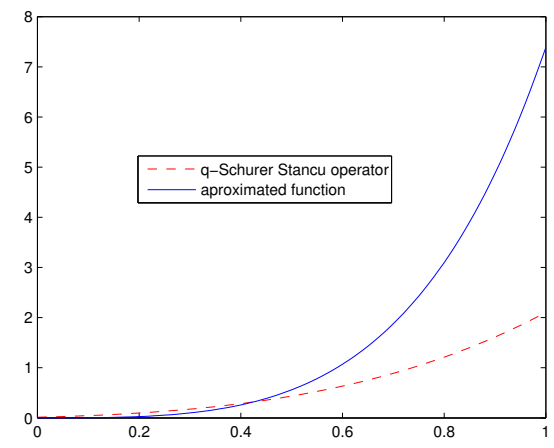

FIGURE 3. The $q$-Schurer-Stancu operators for increasing function and $\beta>\alpha+q^{m}[p]_{q}$

Example 3. If $n=50, p=5, \alpha=3, q=0.9, \beta=8.0211, f(x)=e^{-x^{2}}$, it follows $\tilde{S}_{m, p}^{(\alpha, \beta)}(f, q, x) \geq f(x)$ for all $x \in[0.375,1]$.

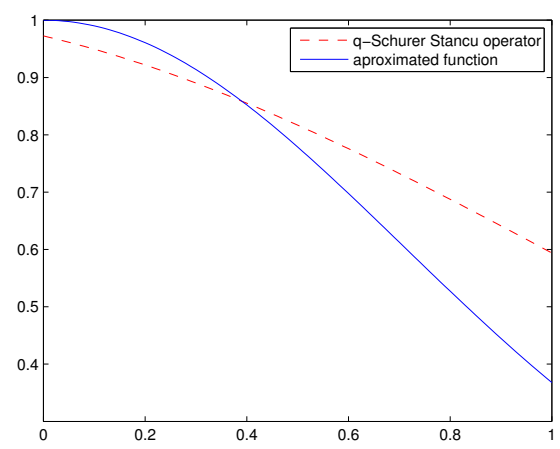

FIGURE 4. The $q$-Schurer-Stancu operators for decreasing function

\section{ACKNOWLEDGMENT.}

Project financed from Lucian Blaga University of Sibiu research grants LBUSIRG-2015-01, No.2032/7. 


\section{REFERENCES}

[1] T. Acar and A. Aral, "On pointwise convergence of q-bernstein operators and their q-derivatives," Numer. Funct. Anal. Optim., vol. 36, no. 3, pp. 287-304, 2015.

[2] A. Acu, "Stancu-schurer-kantorovich operators based on q-integers," Appl. Math. Comput., vol. 259, pp. 896-907, 2015.

[3] O. Agratini, "On a q-analogue of stancu operators," Central European Journal of Mathematics, vol. 8, no. 1, pp. 191-198, 2010.

[4] O. Agratini and C. Radu, "An extension based on qr-integral for a sequence of operators," Appl. Math. Comput., vol. 218, pp. 140-147, 2011.

[5] P. Agrawal, V. Gupta, and A. Kumar, "On q analogue of bernstein-schurer-stancu operators," Appl. Math. Comput., vol. 219, pp. 7754-7764, 2013.

[6] A. Aral and T. Acar, "Voronovskaya type result for q-derivative of q-baskakov operators," J. Appl. Funct. Anal., vol. 7, no. 4, pp. 321-331, 2012.

[7] A. Aral, V. Gupta, and R. P. Agarwal, Applications of $q$ Calculus in Operator Theory. Springer, 2013.

[8] D. Barbosu, "Scurer-stancu type operators," Stud. Univ. Babeş-Bolyai Math., vol. XLVIII, no. 3, pp. 31-35, 2003

[9] O. Dalmanoglu, "Approximation by kantorovich type q-bernstein operators," in 12th WSEAS Int. Conf. on Applied Mathematics, Cairo, Egypte, 2007, pp. 29-31.

[10] O. Dalmanoglu and O. Dogru, "On statistical approximation properties of kantorovich type qbernstein operators," Mathematical and Computer Modelling, vol. 52, pp. 760-771, 2010.

[11] P. Davis, Interpolation and Approximation. New York: Dover Publications Inc., 1976.

[12] A. Lupas, "A q-analogue of the bernstein operator," Seminar on Numerical and Statistical Calculus, University of Cluj-Napoca, vol. 9, pp. 85-92, 1987.

[13] N. Mahmudov, "The moments for q-bernstein operators in the case $0<q<1$," Numer. Algorithms, vol. 53, pp. 439-450, 2010.

[14] C. Muraru, "Note on q-bernstein-schurer operators," Stud. Univ. Babeş-Bolyai Math., vol. 56, no. 2, pp. 489-495, 2011.

[15] H. Oruc and G. Philips, "A generalization of the bernstein polynomials," Proc. Edinb. Math. Soc., vol. 42, pp. 403-413, 1999.

[16] G. Philips, "On generalized bernstein polynomials," in Numerical Analysis, D. Griffits and G. Watson, Eds., 1996, pp. 263-269.

[17] D. Sofonea, "On a q-analogue of a. lupaş operators," in Mathematical Analysis and Approximation Theory, Proceedings of RoGer 2004, Băişoara, I. Gavrea and M. Ivan, Eds. Cluj-Napoca: Mediamira Science Publisher, 2005, pp. 211-223.

[18] V. Videnskii, Linear positive operators of finite rank. Leningrad: A. I. Gerzen State Pedagocical Institute, 1985. 
Authors' addresses

Ana Maria Acu

Lucian Blaga University of Sibiu, Department of Mathematics and Informatics, Str. Dr. I. Ratiu, No.5-7, RO-550012 Sibiu, Romania

E-mail address: acuana77@yahoo.com

\section{Carmen Violeta Muraru}

Vasile Alecsandri University of Bacău, Department of Mathematics, Informatics and Educational Sciences, Calea Mărăşeşti 158, RO-600115 Bacău, Romania

E-mail address: carmen_7419@yahoo.com, cmuraru@ub.ro

Voichiţa Adriana Radu

Babes-Bolyai University, FSEGA, Department of Statistics-Forecasts-Mathematics, Str. Teodor Mihali, No.58-60, RO-400591 Cluj Napoca, Romania

E-mail address: voichita.radu@econ.ubbcluj.ro, voichita.radu@gmail.com 